when memantine was administered at a dose indicated for neuroprotection in rats (20 mg/kg body weight) it induced mild neurotoxicity confined to the retrosplenial cortex. Coadministration of memantine $(10-30 \mathrm{mg} / \mathrm{kg}$ ) and donepezil $(2.5-10 \mathrm{mg} / \mathrm{kg}$ ) substantially increased this neurotoxicity, causing a widespread neurotoxic reaction that killed neurons in many regions of the brain. On the basis of these results, the authors question the use of this drug combination in Alzheimer's disease, especially in the absence of evidence that the combination is beneficial, or that either drug arrests or reverses the disease process.

Original article Creeley CE et al. (2006) Donepezil markedly potentiates memantine neurotoxicity in the adult rat brain. Neurobiol Aging [doi: 10.1016/j.neurobiolaging.2006.10.020]

\section{Automated MRI measurements accurately identify clinically probable Alzheimer's disease}

To date, studies attempting to discriminate patients with Alzheimer's disease (AD) from healthy controls on the basis of brain MRI scans have reported a wide range of accuracy rates. Lerch et al. have suggested that in some cases poor experimental design, small sample sizes and failure to use independent validation cohorts have confounded these results. In response, they have evaluated the capacity of fully automated MRI measurements of cortical thickness to discriminate patients diagnosed with probable $A D(n=19)$ from healthy elderly controls $(n=17)$.

The discriminatory ability of the cortical thickness measurements varied depending on the region of the brain under scrutiny. Areas of the brain classically associated with $A D$ had the best predictive capacity ( $>90 \%$ accuracy for the medial temporal lobes and other limbic structures), whereas the primary motor cortex had the poorest accuracy. Multivariate discriminant analysis of 25 cortical structures revealed $100 \%$ accuracy with 6 different 2structure combinations, all of which included the parahippocampal gyrus. All validation was performed internally, using a jack-knife or leave-one-out approach to prevent overtraining of the discriminant models.

The authors conclude that automated MRI measurements of cortical thickness could be used to improve the clinical diagnosis of probable AD. They suggest that further evaluation of advanced computational techniques applied to MRI in patients with mild cognitive impairment or very early $A D$ would enable imaging to be used as a diagnostic tool across the clinical spectrum of dementia.

Original article Lerch JP et al. (2006) Automated cortical thickness measurements from MRI can accurately separate Alzheimer's patients from normal elderly controls. Neurobiol Aging [doi: 10.1016/j.neurobiolaging.2006.09.013]

\section{Symptoms of depression are an independent risk factor for stroke in the under 65s}

Evidence exists to indicate a possible association between depression and the risk of stroke, and studies have shown a link between depression and increased rates of cardiovascular disease. In a new study, researchers investigated whether depression is associated with an increased risk of stroke and transient ischemic attack (TIA).

Salaycik et al. carried out a prospective study of 4,120 participants from the Framingham Heart Study cohort (mean age 63.9 years; $56 \%$ women). They undertook a baseline evaluation of participants' depressive symptoms, and followed participants for up to 8 years. Continuous monitoring for cerebrovascular events was undertaken, and possible stroke symptoms were assessed by a minimum of two neurologists.

A total of 144 incident strokes $(22$ hemorrhagic and 122 ischemic) and 84 TIAs were reported in 102 men and 126 women during the follow-up period. Separate analyses were performed for those participants aged $<65$ years, and those aged $\geq 65$ years. Among participants under the age of 65 years, the risk of stroke or TIA was 4.21 times higher $(P<0.001)$ for those with symptoms of depression compared with no depressive symptoms; similar results were obtained after adjustment for known stroke risk factors. Among those aged $\geq 65$ years, depressive symptoms were not associated with a significantly higher risk of stroke or TIA.

The authors suggest that an increased awareness of stroke risk in individuals under the age of 65 years with symptoms of depression might allow improved primary prevention of stroke; they propose that the 